https://doi.org/10.22319/rmcp.v10i4.4763

Nota de investigación

\title{
Presencia de hidrocarburos aromáticos policíclicos (HAP) en leche comercializada en la Ciudad de México, evaluando diferentes métodos de extracción
}

Javier Chay Rincón ${ }^{\text {a }}$

José Jesús Pérez González ${ }^{\text {a }}$

Beatriz Sofía Schettino Bermúdez ${ }^{\text {a }}$

Rey Gutiérrez Tolentino ${ }^{\text {a }}$

Dayana Sosa Pacheco ${ }^{\mathrm{b}}$

Arturo Escobar Medina a,b*

Salvador Vega y León ${ }^{a}$

a Universidad Autónoma Metropolitana. Departamento de Producción Agrícola y Animal. Calzada del Hueso 1100, Col. Villa Quietud, Delegación Coyoacán, 04960, Ciudad de México, México.

${ }^{\mathrm{b}}$ Centro Nacional de Sanidad Agropecuaria (CENSA), San José de las Lajas, Mayabeque, Cuba.

*Autor de correspondencia: arturo_c_escobar2002@yahoo.com

\section{Resumen:}

En la actualidad la presencia de hidrocarburos aromáticos policíclicos (HAP) en la leche se reconoce como contaminantes emergentes, por el efecto que pueden provocar en la salud humana. La identificación y cuantificación de estos requiere métodos analíticos que permitan una mayor estimación, por ello el objetivo del presente trabajo fue determinar la presencia de estos compuestos en marcas de leche evaluando tres procedimientos de extracción. Los HAPs por analizar fueron los 16 considerados como prioritarios por la Agencia de Protección 
del Medio Ambiente de los Estados Unidos. Se utilizaron cuatro marcas comerciales de leche entera (tres de leche ultrapasteurizada (UHT) y una de leche pasteurizada (HTST)), cada marca tuvo tres muestreos para una $\mathrm{n}=12$. El muestreo se realizó en el periodo marzo-junio de 2016. Los procedimientos de extracción fueron: saponificación de la leche (variante A), extracción por solución detergente (variante B) y extracción líquida-líquida (variante C); los procedimientos se analizaron por cromatografía de gases con detector de ionización de flama. El $75 \%$ de las muestras fueron positivas a por lo menos uno de los 16 HAP analizados. Los procedimientos de extracción presentaron diferencias entre variantes, la variante A únicamente identificó compuestos de bajo peso molecular (BPM), por su parte, la variante B identificó compuestos de alto peso molecular (APM) y BPM; sin embargo, los resultados muestran que la variante $\mathrm{C}$ presentó mejores porcentajes de recuperación $(58.7 \pm 12.3$ y $81.8 \pm 8.0)$ para HAP de alto y bajo peso molecular que la variante B (15.0 \pm 8.0 y $58.0 \pm 21.0)$ respectivamente.

Palabras clave: Hidrocarburos aromáticos policíclicos, Métodos de extracción, Leche, Cromatografía de gases.

Recibido: 04/02/2018

Aceptado: 21/09/2018

Los hidrocarburos aromáticos policíclicos (HAP) son un grupo de más de 100 sustancias químicas diferentes, que se forman durante la combustión incompleta de la materia orgánica y son liberados al ambiente en grandes cantidades ${ }^{(1,2)}$. Debido a su persistencia y toxicidad la Agencia de Protección del Medio Ambiente de los Estados Unidos (EPA) ha incluido a 16 de estos HAP (Cuadro 1) en su lista de contaminantes orgánicos persistentes ${ }^{(3)}$. 
Cuadro 1. Hidrocarburos aromáticos policíclicos (HAP) de acuerdo a su peso molecular, según la Agencia de Protección Ambiental de los Estados Unidos (EPA)

\begin{tabular}{lll}
\hline HAP & Abreviación & \multicolumn{1}{c}{$\begin{array}{c}\text { Peso molecular } \\
\text { (g/mol) }\end{array}$} \\
\hline Bajo peso molecular (BPM) & & 128 \\
Naftaleno & NAP & 154 \\
Acenafteno & ANA & 152 \\
Acenaftileno & ANY & 166 \\
Fluoreno & FLU & 178 \\
Fenantreno & PHE & 178 \\
Antraceno & ANT & \\
Alto peso molecular (APM) & & 202 \\
Fluoranteno & FLT & 202 \\
Pireno & PYR & 228 \\
Benzo(a)antraceno & BaA & 228 \\
Criseno & CHR & 252 \\
Benzo(b)fluoranteno & BbF & 252 \\
Benzo(k)fluoranteno & BkF & 252 \\
Benzo(a)pireno & BaP & 276 \\
Benzo(g,h,i)perileno & BPE & 276 \\
Indeno (1,2,3-cd)pireno & IPY & 278 \\
Dibenzo(a,h)-Antraceno & DBA & \\
\hline & &
\end{tabular}

EPA, $1998^{(3)}$.

La presencia de estos compuestos en el aire en forma de material particulado fue informada a nivel global $^{(4)}$, y también sus depósitos y acumulación en suelos y pastos ${ }^{(5,6)}$. Cuando los pastos son consumidos por vacas en estado de lactación, se ha demostrado su presencia en leche y derivados lácteos ${ }^{(7-11)}$. La contaminación de leche con HAP, depende de factores ambientales, como son: la fuente de exposición, la etapa de lactancia, el estado de salud del animal y del sistema de $\operatorname{crianza}^{(12,13)}$.

El consumo de leche con HAP representa un riesgo para la salud humana por lo que la Unión Europea (UE), ha establecido un máximo de residuos para la presencia de benzo(a)pireno (BaP) y la sumatoria de cuatro de ellos: $\mathrm{BaP}$, benzo(a)antraceno $(\mathrm{BaA})$, benzo(b)fluoranteno $(\mathrm{BbF})$ y criseno (CHR) en diferentes alimentos que oscila entre 1 a $35 \mu \mathrm{g} \mathrm{kg}$ de grasa ${ }^{(14)}$.

No existe un método oficial para la determinación de HAP en leche, existiendo dos tendencias en la identificación y cuantificación de los mismos: 1) por cromatografía gaseosa empleando detector de ionización de flama y espectrometría de masas ${ }^{(15,16)}$, 2) por cromatografía de líquidos de alta resolución con detector de fluorescencia ${ }^{(7,8,17)}$. Para obtener 
mejores resultados en la preparación de las muestras se han empleado diferentes procedimientos que incluyen saponificación, extracción líquido-líquido (LLE) y limpieza por cromatografía en columna o más recientemente, extracción en fase sólida (SPE) ${ }^{(18,19,20)}$.

La determinación directa de HAP en leche mediante saponificación y extracción posterior, o la extracción de la grasa seguida de purificación muestran perfiles diferentes de los HAP en leche, con un predominio de fenantreno (PHE), antraceno (ANT), fluoreno (FLU), pireno (PYR), BaA y CHR. Por lo anterior el objetivo del presente trabajo fue determinar la presencia de HAP en marcas de leche evaluando tres procedimientos de extracción.

Se seleccionaron de manera aleatoria cuatro marcas comerciales de leche entera (tres ultrapasteurizadas (UHT) y 1 pasteurizada (HTST); cada marca tuvo tres muestreos para una $\mathrm{n}=12$; las muestras se colectaron durante el periodo marzo-junio del 2016 en supermercados ubicados en la Ciudad de México en la delegación de Coyoacán. Las muestras de leche UHT y pasteurizada se almacenaron en el laboratorio de análisis instrumental de la Universidad Autónoma Metropolitana-Xochimilco, las primeras en un lugar seco y fresco, la pasteurizada en refrigeración a $5{ }^{\circ} \mathrm{C}$ hasta su análisis, el cual no superó los cinco días después de su compra. Las muestras se homogeneizaron a baño maría $\left(40^{\circ} \mathrm{C}\right)$ por $30 \mathrm{~min}$ y agitadas manualmente cada 5 min antes de iniciar el proceso de extracción.

Variante A: Saponificación de la leche. De acuerdo al método de Girelli et al ${ }^{(17)}$ modificado: a $4 \mathrm{ml}$ de leche $(4 \mathrm{~g})$ se le agregaron $8 \mathrm{ml}$ de una solución de hidróxido de sodio en etanol $(0.4 \mathrm{M})$; la mezcla se homogeneizó por un minuto en vortex y se colocó en un baño térmico a $40{ }^{0} \mathrm{C}$ hasta casi sequedad $(1 \mathrm{ml})$. Se llevó a sequedad bajo una corriente de nitrógeno. Finalmente se reconstituyó en 1,000 $\mu \mathrm{l}$ de isooctano y se almacenó a $-20{ }^{0} \mathrm{C}$ hasta su análisis.

Variante B: Extracción con solución detergente. En un matraz volumétrico de $500 \mathrm{ml}$ se adicionaron $250 \mathrm{ml}$ de muestra más $250 \mathrm{ml}$ de una solución detergente $(50 \mathrm{~g}$ de hexametafosfato de sodio en $24 \mathrm{ml}$ de Tritón X -100 disueltos en un litro de agua). El matraz se agitó vigorosamente colocándose en un baño de agua a $90{ }^{\circ} \mathrm{C}$ invirtiéndose cada 15 min hasta lograr una separación en el cuello del matraz de la materia grasa. La grasa extraída se filtró a $50{ }^{\circ} \mathrm{C}$ a través de un papel filtro Whatman número 4 , en presencia de sulfato de sodio anhidro y se conservó en tubos de vidrio a $-20{ }^{\circ} \mathrm{C}$ hasta su análisis ${ }^{(21)}$.

Variante C: Extracción líquida-líquida (AOAC 989.05). En un embudo de separación se adicionaron $150 \mathrm{ml}$ de muestra y $0.5 \mathrm{~g}$ de ácido etilendiaminotetraacético (EDTA), agitándose por un minuto y se reposó por $2 \mathrm{~min}$. Se adicionaron $50 \mathrm{ml}$ de metanol, volviéndose a agitar por un minuto. Se repitió la operación, adicionándose $50 \mathrm{ml}$ de éter dietílico y $50 \mathrm{ml}$ de éter de petróleo. Se dejó en reposo hasta la separación de la fase orgánica (sobrenadante), se drenó la capa inferior y el sobrenadante, pasándose por papel filtro Whatman número 1 , adicionando $5 \mathrm{~g}$ de sulfato de sodio anhidro. La capa orgánica se rotovaporó a $40{ }^{\circ} \mathrm{C}$ y se traspasó a un frasco de $5 \mathrm{ml}$ y fue guardado a $-20{ }^{0} \mathrm{C}$ hasta su análisis. 
La muestra de grasa (saponificada) se depositó lentamente sobre una columna que contenía $6 \mathrm{~g}$ de sílica gel (en la parte inferior) y $1 \mathrm{~g}$ de sulfato de sodio anhidro. Se adicionaron $20 \mathrm{ml}$ de hexano, la fase orgánica se marcó como F1. Se cambió el matraz y se adicionaron $30 \mathrm{ml}$ de hexano-diclorometano 9:1 v/v, se dejó correr lentamente y al llegar al ras del sulfato de sodio se adicionaron $20 \mathrm{ml}$ de hexano-diclorometano 1:1 v/v. Toda la fase orgánica se colectó en un solo matraz y se marcó como F2 (hidrocarburos aromáticos policíclicos recobrados). El extracto $\mathrm{F} 2$ se rotovaporó a $40{ }^{\circ} \mathrm{C}$ hasta casi sequedad $(1 \mathrm{ml})$. La muestra se traspasó a un vial color ámbar, se llevó a sequedad bajo una corriente de nitrógeno, se reconstituyó en 250 $\mu l$ de isooctano y se almacenó a $-20{ }^{\circ} \mathrm{C}$ hasta su análisis ${ }^{(19)}$.

Se utilizó un cromatógrafo de gases digital de alta resolución modelo Shimadzu GC 2010 con automuestreador, un inyector PTV a $250{ }^{\circ} \mathrm{C}$ en modo Splitless con un sampling time (1 min), purga Flow $5.0 \mathrm{ml} \mathrm{min}^{-1}$, Septum purga $5 \mathrm{ml} \mathrm{min}^{-1}$. Como gas transportador se usó nitrógeno a un flujo de $9.8 \mathrm{ml} \mathrm{min}^{-1}$. Se trabajó con una columna HP5-MS (30 m x $0.025 \mathrm{~mm}$ DI. X $0.25 \mu \mathrm{m}$ de grosor). El programa de temperatura del horno se estableció de la siguiente manera: temperatura inicial de $40{ }^{\circ} \mathrm{C}$ por $3 \mathrm{~min}$, luego un incremento de $2{ }^{\circ} \mathrm{C} / \mathrm{min}$ hasta los $50{ }^{\circ} \mathrm{C}$, de los $50{ }^{\circ} \mathrm{C}$ a los $160{ }^{\circ} \mathrm{C}$ con un aumento de $3{ }^{\circ} \mathrm{C} / \mathrm{min}$, de los $160{ }^{\circ} \mathrm{C}$ a los $210{ }^{\circ} \mathrm{C}$ con un aumento de $5{ }^{\circ} \mathrm{C} / \mathrm{min}$, de 210 a $255^{\circ} \mathrm{C}$ con un incremento de $7{ }^{\circ} \mathrm{C} / \mathrm{min}$, de 255 a $265{ }^{\circ} \mathrm{C}$, con un aumento de $4{ }^{\circ} \mathrm{C} / \mathrm{min}$. Por último, de 265 a $300{ }^{\circ} \mathrm{C}$ un incremento de $5{ }^{\circ} \mathrm{C} / \mathrm{min}$, manteniéndose por 5 min. Para el análisis de los cromatogramas se empleó el Sotware GG solution.

Se empleó un cromatógrafo Agilent GC 5890, se aplicó $1 \mu \mathrm{l}$ de extracto de la muestra mediante inyección (en columna). Se utilizó columna capilar Rtx-5Sil MS (30 m, x 0.25 mm DI, $0.25 \mu \mathrm{m}$ de grosor) de Restek (Bellafonte, PA, EE. UU.) y una precolumna Siltek de $2 \mathrm{~m}$ ( $0.53 \mathrm{~mm}$ DI.) del mismo proveedor. Se usó helio como gas transportador a un flujo constante de $1 \mathrm{ml} / \mathrm{min}$. La temperatura del inyector se estableció en $3{ }^{\circ} \mathrm{C}$ superior a la temperatura del horno en todo momento. La temperatura del horno se programó de la siguiente manera: 1 min a $100{ }^{\circ} \mathrm{C}$, de $100{ }^{\circ} \mathrm{C}$ a $300^{\circ} \mathrm{C}$ a $5{ }^{\circ} \mathrm{C} /$ min y 15 min a $300{ }^{\circ} \mathrm{C}$. La detección de los analitos se realizó con un equipo Agilent MS 5972 en el modo de impacto de electrones con una energía de ionización de 70 eV y monitorización de ión único ${ }^{(22)}$ (Figura 1). 
Figura 1: Cromatograma de espectrometría de masas, identificando un pico de la muestra para saber si corresponde a un compuesto nativo

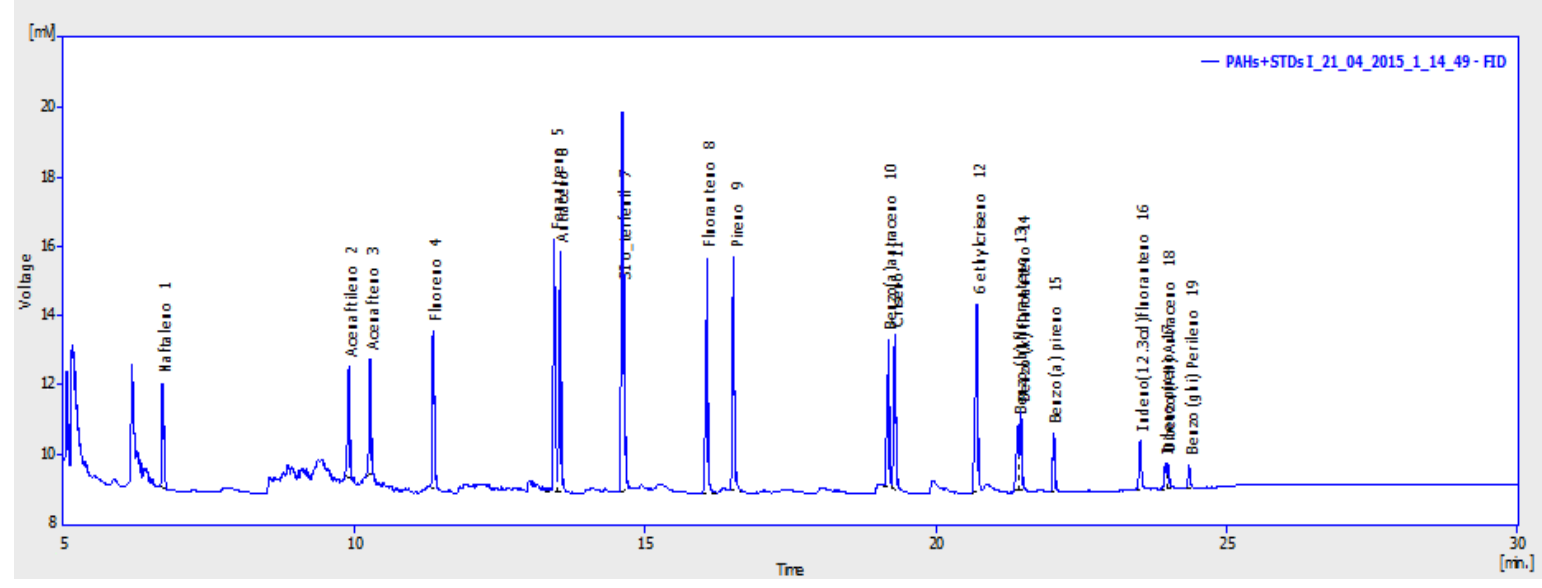

Estándar interno: ortoterphenyl (pico 7); 6 ethyl chrysene (pico 12); indeno[1,2,3-cd]fluoranthene (pico 16).

Los reactivos químicos y solventes fueron de calidad reactivo y HPLC respectivamente, de la firma comercial J. T. Baker chemical, USA. Para la detección y cuantificación de los analitos, se utilizó una mezcla de patrones con 16 compuestos de HAP recomendados en el método EPA 610 (Chemicalservice, USA), a saber: naftaleno (NAP); acenaftaleno (ALC); acenaftileno (ACY); fluoreno (FLU); fenantreno (PHE); antraceno (ANT); fluoroantraceno (PMA); pireno (PYR); benzo(a)antraceno (BaA); criseno (CHR); benzo(b)fluoranteno $(\mathrm{BbF})$; benzo(k)fluoranteno $(\mathrm{BkF})$; benzo(a)pireno $(\mathrm{BaP})$; dibenzo(ab)antraceno (DBA); benzo(ghi)perileno (BGP) e indeno (cd)pireno (IcdPy) (Cuadro 1).

La extracción por medio de la saponificación de la leche (variante A) ${ }^{(17)}$ identificó solamente HAP de BPM, mientras que la extracción con solución detergente (variante B) ${ }^{(19)}$ logró determinar HAP de BPM y APM. Para el método B estas últimas moléculas representaron el $33.33 \%$ de la concentración total de HAP, en tanto que, compuestos de BPM representaron el $66.66 \%$ (Cuadro 2). 
Cuadro 2: Concentración $\left(\mu \mathrm{g} \mathrm{g}^{-1}\right)$ de hidrocarburos aromáticos policíclicos (HAP) en leche por dos métodos de extracción

\begin{tabular}{lll}
\hline HAP & Método A & Método B \\
\hline NAP & 0.066 & $\mathrm{Nd}$ \\
ALC & 0.200 & 0.372 \\
ACY & 0.066 & $\mathrm{Nd}$ \\
FLU & $\mathrm{Nd}$ & 0.915 \\
PHE & $\mathrm{Nd}$ & 7.153 \\
ANT & 5.385 & 14.924 \\
FLT & $\mathrm{Nd}$ & $\mathrm{Nd}$ \\
PYR & $\mathrm{Nd}$ & 3.773 \\
BaA & $\mathrm{Nd}$ & $0-.056$ \\
CHR & $\mathrm{Nd}$ & 0.044 \\
BbF & $\mathrm{Nd}$ & 1.264 \\
BkF & $\mathrm{Nd}$ & 0.750 \\
BaP & $\mathrm{Nd}$ & $0-.114$ \\
DBA & $\mathrm{Nd}$ & 4.061 \\
BGP & $\mathrm{Nd}$ & $\mathrm{Nd}$ \\
IcdPy & $\mathrm{Nd}$ & 1.641 \\
Suma de 16 HAPs & 5.717 & 35.067 \\
Suma de 4 HAPs & 0.0 & 1.478 \\
Suma de HAPs DE BPM & $5.717(100 \%)$ & $23.365(66.6 \%)$ \\
Suma de HAPs DE APM & 0.00 & $11.702(33.4 \%)$ \\
\hline MÉ & &
\end{tabular}

Métodos= A: saponificación y extracción directa; método B: extracción con solución detergente. $\mathrm{Nd}=$ no determinado; $\mu \mathrm{g} \mathrm{g}^{-1}$ : microgramo de HAP por gramo de grasa láctea.

En este estudio la extracción de HAP por saponificación de la leche (variante A), difiere de otros estudios que muestran un predominio de HAP de APM con mayores concentraciones de PHE y ANT, así como la relación HAP de BPM y total de HAP que se ha reportado entre 50 y $68 \%{ }^{(17,23)}$. La ausencia de compuestos de APM en el método A, puede deberse a que las muestras analizadas presentan bajas concentraciones, aspecto que ha sido mencionado por otros autores ${ }^{(24)}$, por otra parte el empleo de $4 \mathrm{ml}$ de muestras de leche, no es suficiente para sobrepasar el límite de detección de los HAP en nuestras condiciones (detector de ionización de flama).

Fórmulas lácteas para infantes ${ }^{(18,25)}$, leche entera y $\mathrm{UHT}^{(17)}$ han reportado concentraciones bajas de HAP de APM. Por otra parte, compuestos de BPM (2 y 3 anillos) en particular NAP, ACE y ACY no se informan en diversos estudios ${ }^{(9,17,23)}$, ya que reportan porcentajes de 
recuperación menores a 50, posiblemente por su alta volatilidad ${ }^{(17)}$. Sin embargo, es probable que el tiempo y la temperatura en la que se realiza la saponificación, jueguen un papel importante en los recobrados del mismo, en este estudio, se aplicó una temperatura de $40{ }^{\circ} \mathrm{C}$ pudiendo detectar los compuestos de BPM, similar a cuando se realiza la saponificación a 60 ${ }^{\circ} \mathrm{C}^{(24)}$, mientras la saponificación a $80^{\circ} \mathrm{C}$, solo detecta PHE y $\mathrm{ANT}^{(23)}$, lo que es evidencia, que la temperatura de saponificación es un punto crítico en la determinación de $\operatorname{HAP}^{(9)}$.

A diferencia del método anterior, cuando se emplea la grasa sin saponificar y se pasa a una columna para su purificación, los resultados obtenidos permiten identificar HAP de BPM y APM, en valores de 66.6 y $33.4 \%$ respectivamente, con perfiles similares a cuando se estudiaron 31 muestras de leche procedente de Brasil y Argentina con valores promedios de 75.5 y $24.5 \%$ para HAP de BPM y APM respectivamente ${ }^{(7)}$, mientras que en leche fresca procedente de granjas cercanas a una área industrial se obtuvieron valores de HAP de BPM que oscilaron entre 40 y $69 \%{ }^{(19)}$; las diferencias encontradas pueden estar relacionadas con la forma de extracción de la grasa láctea, que se fundamentan en una extracción con solventes orgánicos $^{(7)}$ y otra con una solución detergente ${ }^{(19)}$.

La mayor parte de los estudios que emplean la saponificación directa en las muestras usan detectores acoplados a masas o detectores fluorescentes, lo que les permite cuantificar bajas concentraciones de HAP en las muestras ${ }^{(17,18,24)}$; sin embargo, para alcanzar la sensibilidad adecuada, cuando se usa cromatografía de gases con detección de ionización de flama, se requiere emplear una mayor cantidad de grasa láctea en la determinación, aspecto que es posible obtener por el uso de una solución detergente, aunque los HAP se pueden perder por efecto de la temperatura a la que se someten las muestras durante la extracción $\left(90^{\circ} \mathrm{C}\right)$.

En el Cuadro 3 se presentan los porcentajes de recuperación de HAP en las muestras analizadas de acuerdo a los métodos B y C y la confirmación por CG-MS. Los recobrados obtenidos muestran una variabilidad alta, en función de la clasificación de los HAP de BPM y APM, donde los recobrados mayores se obtienen para los de APM. La variabilidad de los recobrados entre BPM y APM está asociados con el tipo de extracción de la grasa y la temperatura en la rotovaporación. En las condiciones de este trabajo, el método más adecuado fue el método $\mathrm{C}$, ya que alcanza recobrados que varían entre 45.3 a $95.1 \%$, similares a los encontrado en otro estudio donde se emplearon solventes orgánicos en la extracción de la grasa, reportando valores de recobrados entre 40 y $125 \%$ sin especificar los compuestos $^{(26)}$. Otro estudio, pero en leche humana el recobrado osciló entre 42 y $101 \%$ justificando los mismos con su punto de ebullición encontrando una $R^{2}$ de $0.779^{(27)}$. Un estudio en leche de polvo, pero empleando un baño de ultrasonido y posterior purificación en columna, los recobrados oscilaron entre 95 y $98 \%{ }^{(15)}$; lo mismo sucede cuando se emplea un sistema de microextracción de fase sólida donde los recobrados oscilaron entre 87.6 y $112 \%$. 
Cuadro 3: Porcentajes de recuperación de hidrocarburos aromáticos policíclicos (HAP) en leche empleando dos métodos de extracción (media \pm error estándar)

\begin{tabular}{lll}
\hline HAP & Método B & Método C \\
\hline NAP & Nd & Nd \\
ALC & $15.2 \pm 7.3$ & $45.3 \pm 19.0$ \\
ACY & $10.8 \pm 9.1$ & $46.5 \pm 14.7$ \\
FLU & $23.8 \pm 4.8$ & $72.3 \pm 20.9$ \\
PHE & $28.3 \pm 10.7$ & $67.6 \pm 22.6$ \\
ANT & $30.4 \pm 15.3$ & $61.6 \pm 16.9$ \\
FLT & $48.0 \pm 10.9$ & $77.5 \pm 24.8$ \\
PYR & $44.7 \pm 1467$ & $72.0 \pm 25.9$ \\
BaA & $70.9 \pm 16.7$ & $80.0 \pm 14.4$ \\
CHR & $59.3 \pm 15.5$ & $95.1 \pm 27.5$ \\
BbF & $93.5 \pm 21.1$ & $80.9 \pm 11.3$ \\
BkF & $45.4 \pm 11.1$ & $72.7 \pm 22.4$ \\
BaP & $127.0 \pm 35.0$ & $85.6 \pm 7.0$ \\
DBA & $78.4 \pm 17.9$ & $92.1 \pm 18.3$ \\
BGP & $64.5 \pm 15.7$ & $86.9 \pm 21.9$ \\
IcdPy & $66.3 \pm 14.7$ & $75.0 \pm 15.7$ \\
Suma de HAPs de BPM & $15 \pm 8 \%$ & $58.7 \pm 12.3$ \\
Suma de HAPs de APM & $58 \pm 21 \%$ & $81.8 \pm 8.0$ \\
\hline
\end{tabular}

Métodos= B: extracción con solución detergente, método C: extracción líquida-líquida.

Se demuestra que la forma de extracción de la grasa constituye un punto crítico en la determinación de HAP en muestras de leche, sin embargo, los recobrados encontrados en el método $\mathrm{C}$, están acordes cuando se determinan contaminantes ambientales en matrices biológicas y aparecen en concentraciones inferiores a $1 \mu \mathrm{g} \mathrm{kg}^{-1}$ donde su intervalo puede ser desde $-50 \%$ a $+20 \%{ }^{(29)}$, lo cual permite evaluar la presencia de HAP en muestras de leche con una exactitud adecuada.

El Cuadro 4 presenta la incidencia, sumatoria, valor máximo y mínimo de cada HAP en el total de muestras analizadas. Una de las marcas no presentó HAP, mientras que en el resto se detectaron al menos uno de los 16 compuestos, es decir, $75 \%$ de las muestras fueron positivas a la presencia de HAP. Los compuestos con mayor incidencia en las muestras fueron PHE y ANT con $54.5 \%$ y FLUO y DBA con $45.5 \%$, donde el ANT presentó la mayor concentración (341 $\left.\mu \mathrm{g} \mathrm{g}^{-1}\right)$ seguido del PHE $\left(20 \mu \mathrm{g} \mathrm{g}^{-1}\right)$ y el DBA $\left(12.3 \mu \mathrm{g} \mathrm{g}^{-1}\right)$. Estos resultados coinciden con otros autores donde compuestos de BPM aparecen con mayor frecuencia y concentración ${ }^{(17,23)}$. 
Cuadro 4: Presencia de hidrocarburos aromáticos policíclicos (HAP) en las muestras de leche analizadas $(\mathrm{n}=12)$

\begin{tabular}{lllllllllllll}
\hline & ACE & FLUO & PHE & ANT & PYR & BaA & CHR & BbF & BkF & BaP & IND & DBA \\
\hline \% Inc & 36.4 & 45.5 & 54.5 & 54.5 & 9.1 & 27.3 & 27.3 & 27.3 & 18.2 & 9.1 & 27.3 & 45.5 \\
Suma & 1.2 & 5.6 & 20.0 & 341.0 & 3.8 & 0.2 & 0.2 & 2.3 & 1.1 & 0.1 & 6.3 & 12.3 \\
Min & 0.2 & 0.3 & 0.4 & 0.0 & 3.8 & 0.0 & 0.0 & 0.2 & 0.3 & 0.1 & 0.7 & 0.1 \\
Max & 0.6 & 3.6 & 7.5 & 155.0 & 3.8 & 0.1 & 0.1 & 1.3 & 0.7 & 0.1 & 3.0 & 5.6 \\
\hline
\end{tabular}

Inc= incidencia, Min= mínimo, Max= máximo.

Por otra parte, la marca D presentó la mayor mediana de la sumatoria de 4 HAP (Cuadro 5); esta concentración sobrepasa el valor establecido por la UE que es $1 \mu \mathrm{g} \mathrm{kg}^{-1(14)}$ para fórmulas lactantes, lo que implica un riesgo para la salud humana. Esta misma marca incorpora grasa vegetal en su formulación.

Cuadro 5: Resultados de la mediana de la sumatoria de 16 y 4 hidrocarburos aromáticos policíclicos (HAP) en marcas de leche de mayor consumo en la Ciudad de México

\begin{tabular}{|c|c|c|}
\hline Marcas & $\Sigma 16$ HAPs $\mu \mathrm{g} \mathrm{kg}^{-1}$ & $\Sigma$ 4HAPs $\mu \mathrm{g} \mathrm{kg}^{-1}$ \\
\hline $\mathrm{A}$ & $\mathrm{Nd}$ & $\mathrm{Nd}$ \\
\hline B & 47.56 & 0.23 \\
\hline $\mathrm{C}$ & 93.95 & 1.14 \\
\hline $\mathrm{D}$ & 51.49 & 4.04 \\
\hline
\end{tabular}

Las variantes B y C durante la determinación de los ácidos en muestras de leche presentan variables en los porcentajes de HAP de bajo y alto peso molecular, donde la variante C mostró los mejores recuperados, mientras la variante B puede ser una alternativa cuando se emplea la CG-FID. El $75 \%$ de las muestras de leche fueron positivas a la presencia de HAP, donde el $50 \%$ superaron el valor umbral de la UE. 


\section{Literatura citada:}

1. AOAC 989.05. Fat in milk. Official methods of analysis of AOAC INTERNATIONAL, 18th ed. Editors Horwitz W, Latimer GW editors. 2005.

2. Shen H, Huang Y, Wang R, Zhu D, Li W, Shen G, et al. Global atmospheric emissions of polycyclic aromatic hydrocarbons from 1960 to 2008 and future predictions. Environ Sci Technol 2013;47:6415-6424.

3. EPA. Environment Protection Agency. Locating and estimating air emissions from sources of polycyclic organic matter EPA-454/R-98-014. USA 1998. https://www.epa.gov/ttn/chief/le/pompta.pdf. Accessed Nov 15, 2017.

4. Bogdal C, Scheringer M, Abad E, Abalos M, Van-Bavel B, Hagberg J, et al. Worldwide distribution of persistent organic pollutants in air including results of air monitoring by passive air sampling in five continents. TrAC 2013;46:150-161.

5. Liu W, Wang Y, Chen Y, Tao S, Liu W. Polycyclic aromatic hydrocarbons in ambient air, surface soil and wheat grain near a large steel smelting manufacturer in northern China. J Environ Sci (China) 2017; 57:93-103.

6. Liang J, Fang H, Zhang T, Wang X. Polycyclic aromatic hydrocarbons in the leaves of twelve plant species along an urbanization gradient in Shanghai, China. Environ Sci Pollut Res Int 2017;24(10):9361-9369.

7. García-Londoño AV, Reynoso M, Resnick S. Polycyclic aromatic hydrocarbons in milk powders marketed in Uruguay. Food Addit Contam Part B 2017;10(4):284-291.

8. Fasano E, Esposito F, Scognamiglio G, Cocchieri R, Cirillo T. Detection of polycyclic aromatic hydrocarbons in smoked buffalo mozzarella cheese produced in Campania Region, Italy. J Sci Food Agr 2016;96(5):1704-1708.

9. Battisti C, Girelli AM, Tarola AM. Polycyclic aromatic hydrocarbons (PAHs) in yogurt samples. Food Addit Contam Part B 2015;8(1):50-55.

10. Costera A, Feidt C, Dziurla MA, Monteau F, Le-Bizec B, Rychen G. Bioavailability of polycyclic aromatic hydrocarbons (PAHs) from soil and hay matrices in lactating goats. J Agric Food Chem 2009;57(12):5352-5357.

11. Rychen G, Jurjanz S, Toussaint H, Feidt C. Dairy ruminant exposure to persistent organic pollutants and excretion to milk. In: Faye, Bernard, Sinyavsky, Yuriy edtitors. Impact of pollution on animal products. 1rst ed. Spinger, Netherlands: Springer Science+Bussiness Media BV; 2008:63-83. 
12. Mercogliano R, De-Felice A, Chirollo C, Marrone R, Panzardi M, Vollano L, et al. Contaminazione ambientale da idrocarburi in ricota e mozzarella di bufala. A. I. V. I. 2010;8:45-47.

13. Zanieri L, Galvan P, Checchini L, Cincinelli A, Lepri L, Donzelli GP, et al. Polycyclic aromatic hydricarbons (PAHs) in human milk from Italian women: influence of cigarette smoking and residential area. Chemosphere 2007;67(7):1265-1274.

14. UE. Unión Europea. Amending Regulation (EC) No 1881/2006 about maximum levels for polycyclic aromatic hydrocarbons in foodstuffs. No 835/2011. The European Commission. Official Journal of the European Union 2011; L 215/4.

15. Dobrinas S, Soceanu A, Popescu V, Coatu V. Polycyclic aromatic hydrocarbons and pesticides in milk powder. J Dairy Res 2016;83(2):261-265.

16. Sanagi MM, Loh SH, Wan-Ibrahim WA, Hasan MN, Aboul-Enein HY. Determination of polycyclic aromatic hydrocarbons in fresh milk by hollow fiber liquid -phase microextraction -gas chromatography mass spectrometry. J Chromatogr Sci 2013;51 (2):112-116.

17. Girelli AM, Sperati D, Tarola AM. Determination of polycyclic aromatic hydrocarbons in Italian milk by HPLC with flourescence detection. Food Addit Contam Part A 2014;31(4):703-710.

18. Santonicola S, Albrizio S, Murru N, Ferrante MC, Mercogliano R. Study on the occurrence of polycyclic aromatic hydrocarbons in milk and meat/fish based baby food available in Italy. Chemosphere 2017;184:467-472.

19. Gutierrez R, Vega S, Ortiz R, Pérez JJ, Schettino B. Presence of PAHs in milk of industrial farms from Tizayuca, Hidalgo, Mexico. J Environ Sci Health 2015;50(5):317321.

20. Kim SR, Halden RU, Buckley TJ. Polycyclic aromatic hydrocarbons in human milk of nonsmoking U.S. women. Environ Sci Technol 2008;42(7):2663-2667.

21. Pérez JJ, Vega S, Gutiérrez R, López Y, Faure R, Escobar A. Polycyclic biphenyls (PCBs) residues in milk from an agroindustrial zone of Tuxpan, Veracruz, Mexico. Chemosphere 2012;89(4):404-408.

22. Bucheli TD, Blum F, Desaules A, Gustafsson O. Polycyclic aromatic hydrocarbons, black carbon and molecular markers in soils of Switzerland. Chemosphere 2004;56 (11):1061-1076.

23. Naccari C, Cristani M, Giofrè F, Ferrante M, Siracusa L, Trombetta D. PAHs concentration in heat-treated milk samples. Food Res Int 2011;44 (3):716-724. 
24. Chung TL, Liao CJ, Chen MF. Comparison of liquid -liquid extraction and solid -phase extraction for the determination of polycyclic aromatic hydrocarbons in milk of Taiwan. J Taiwan Inst Chem Eng 2010;41(2):178-183.

25. Kyoung $\mathrm{CH}$, Seung SH. Evaluation of polycyclic aromatic hydrocarbons and risk assessment for infant formula in Korea. Food Sci Biotech 2012;21(5):1329-1334.

26. Grova N, Feidt C, Crepineau C, Laurent C, Lafargue PE, Hachini A, et al. Detection of polycyclic aromatic hydrocarbons levels in milk collected near potencial contamination sources. J Agric Food Chem 2002;50(16):4640-4642.

27. Del-Bubba M, Zanieri L, Galvan P, Donzelli GP, Checchini L, Lepri L. Determination of polycyclic aromatic hydrocarbons (PAHs) and total fats in human milk. Ann Chim 2005;95(9-10):629-641.

28. Aguinaga N, Campillo N, Viñas P, Hernández-Córdoba M. Determination of 16 polycyclic aromatic hydrocarbons in milk and related products using solid-phase microextraction coupled to gas chromatography-mass spectrometry. Anal Chim Acta 2007;596 (2):285-290.

29. UE. Unión Europea. DECISIÓN DE LA COMISIÓN de 12 de agosto de 2002 por la que se aplica la Directiva 96/23/CE del Consejo en cuanto al funcionamiento de los métodos analíticos y la interpretación del resultado. Diario Oficial de las Comunidades Europeas (2002/657/CE) 2002; L 221/8. 29. 